\title{
INOCENCIA Y RAZONAMIENTO PROBATORIO
}

\author{
Innocence and probatory reasoning
}

Jonatan Valenzuela $S .^{*}$

Resumen: el presente trabajo está dirigido a explorar la relación entre la noción institucional de inocencia para el proceso penal y el modo de razonamiento desplegado a propósito de la prueba. La tesis central es que la inocencia sirve de base al modo de razonamiento que el juez debe tener a la hora de adjudicar en el proceso penal suponiendo la aplicación de un determinado estándar de prueba.

Palabras clave: razonamiento probatorio - presunción de inocencia - proceso penal chileno - estándar de prueba.

Abstract: The article explores the relationship between the institutional notion of innocence in the criminal process and the reasoning developed in a probatory context. The central thesis is that the presumption of innocence is the ground upon which the judge reason in the moment of adjudication in criminal process, assuming the application of some standard of proof.

Keywords: probatory reasoning - presumption of innocence - chilean criminal process standard of proof.

\section{Introducción}

En el proceso penal, como en todos los procesos judiciales, existe un intento de tomar decisiones con la mayor información posible. Es claro, a esta altura, que el objetivo epistémico por excelencia del proceso es la averiguación de la verdad y que esa verdad supone una relativa correspondencia con los hechos que han dado lugar al conflicto sometido a decisión de un determinado juez.

Debido a que el proceso está diseñado para la toma de decisiones (la más evidente es la decisión de absolución o condena del acusado), resulta necesario contar con mecanismos que impidan la incertidumbre acerca de los hechos. Un escenario de duda respecto de una determinada proposición de verdad o falsedad

\footnotetext{
* Abogado. Licenciado en Ciencias Jurídicas y Sociales (U. de Chile), Doctor en Derecho (U. de Girona). Profesor de Teoría del Derecho y Derecho Procesal Penal en la Universidad Austral de Chile. Este trabajo es una versión corregida y (espero que) mejorada de la ponencia presentada en el seminario "Prueba, presunciones y presunción de inocencia" organizado por el Centro de Estudios de la Justicia de la Universidad de Chile el día 17 de diciembre de 2012. Agradezco a los asistentes a este seminario los comentarios y sugerencias planteadas. Correo electrónico: valenzuelasaldias@gmail.com.
}

Este artículo fue recibido el 27 de febrero de 2013, siendo aprobada su publicación con fecha 8 de mayo de 2013. 
Valenzuela - Inocencia y razonamiento probatorio

de un determinado enunciado acerca de los hechos impide, por completo, tomar una decisión que suponga dar por concurrente la verdad o falsedad del enunciado acerca de los hechos. En otros términos, podemos decidir acerca de la condena de determinado imputado en la medida que podamos superar la incertidumbre acerca de la culpabilidad o inocencia del imputado, no podemos condenar ni absolver razonablemente a quien consideremos culpable e inocente a la vez en un mismo proceso.

El estatuto de la inocencia es uno de estos mecanismos. La presunción de inocencia (en adelante PI) parece estar en el centro de las intuiciones acerca del proceso, sin embargo, cuenta con una redacción bastante difusa tanto a nivel normativo como en el nivel de sus usos lingüísticos. ${ }^{1}$

Las presunciones son instituciones de uso común entre los juristas. Se suele asociar la expresión presunción a diversas maneras de evitación de incertidumbres en el derecho. Las reglas llamadas presunciones constituyen una manera de negar un determinado déficit de información que nos impida la toma de una determinada decisión. De algún modo, estas reglas nos ponen en una determinada situación por defecto. ${ }^{2}$

Existe una presunción de conocimiento del derecho, en materia contractual se presume la buena fe, el matrimonio y la cohabitación permiten presumir la filiación de un hijo nacido en cierto período, la desaparición de una persona en ciertas circunstancias y concurriendo ciertas condiciones legales permiten decretar su muerte presunta. Todas estas presunciones se encuentran establecidas por el legislador y constituyen una manera de evitar que la falta de información fiable acerca de un hecho en el pasado nos impida tomar una decisión respecto del contrato, la filiación, la muerte, el conocimiento del derecho u otros.

Además, se suele sostener que se presume que todas las personas son inocentes hasta que no se declare, por una vía institucional, su culpabilidad. Este es el caso de la PI contenida en el artículo 4 del CPP: "Presunción de inocencia del imputado. Ninguna persona será considerada culpable ni tratada como tal en tanto no fuere condenada por una sentencia firme."

Como sabemos esta es una institución multidimensional. Aparece en diversas formas durante el proceso, tiene el estatuto de principio informador, de

\footnotetext{
${ }^{1}$ En efecto, las proyecciones de la PI no se encuentran detalladamente en nuestra doctrina, la que parece centrarse en la PI como regla de trato. Sobre la proyecciones de la PI véase Valenzuela, Jonatan: "Presumir responsabilidad: sobre una relación entre la prisión preventiva y la presunción de inocencia en el derecho procesal chileno", en Revista de Estudios de la Justicia, número 14, 2011.

${ }^{2}$ Las presunciones han sido objeto de amplio debate en su vertiente probatoria además, pues suelen ser descritas como reglas que "invierten la carga de la prueba", véase Yablon, Charles: "A Theory of Presumptions”, en Law, Probability and Risk, núm. 2, 2003, pp. 227-236. Ineludible resulta Bohlen, Francis: “The Effect of Rebuttable Presumptions of Law upon the Burden of Proof', en University of Pennsylvania Law Review and American Law Register, vol. 68, núm. 4, 1920.
} 
regla de trato (estas dos resultan evidentes del texto del mismo art. 4), y también tiene la dimensión de regla de prueba y de regla de juicio. Sobre estos dos últimos puntos es sobre los que quiero poner mi atención. ${ }^{3}$

Una cuestión llamativa de la PI es que no genera el mismo tipo de práctica lingǘstica que el resto de nuestros ejemplos. Como se sabe, el uso común para referirnos a alguien de quien parece sospecharse que ha cometido un delito, y es puesto, por ello, en situación de ser imputado o acusado en un proceso es algo así como "el presunto responsable". No obstante, es claro que la carga de probar los hechos que hacen que una determinada persona sea tenida por culpable recae en la acusación y que sin actividad probatoria de cargo esa persona debe ser tenida como inocente.

Todas las presunciones parecen responder a la siguiente estructura:

“a) Un hecho presunto: lo sospechado o conjeturado;

b) Un hecho base: los indicios o señales;

c) Una conexión entre ellos. La conexión entre el hecho base y el hecho presunto presupone una regla de presunción; es decir, un enunciado general cuya aceptación autoriza el paso de uno a otro hecho."

Esta estructura puede verse cuestionada en el caso de la PI. El hecho presunto es, al parecer, que las personas son inocentes. El hecho base es, al parecer, que una determinada persona no es culpable. $\mathrm{O}$ si quiere verse de otro modo, no parece tratarse exactamente de dos hechos sino de uno.

¿Es este un problema relevante a efectos de entender la manera en que la PI se despliega en el ámbito del razonamiento probatorio?

A mi juicio la respuesta es negativa. Parece que nos encontramos ante una formulación normativa que en términos estructurales no merece ser llamada presunción, pero por otro lado sí nos encontramos ante una regla que se despliega como una prescripción a la hora de determinar la carga de la prueba (de producir prueba stricto sensu) y al momento de justificar la decisión judicial.

El objetivo de este trabajo es llamar la atención sobre el sentido probatorio que tienen dos clases de proyecciones de la PI.

\footnotetext{
${ }^{3}$ Sobre estas cuatro formas de ver a la PI puede consultarse el trabajo de Ferrer, Jordi: "Una concepción minimalista y garantista de la presunción de inocencia”, Revista de la Maestría en Derecho Procesal, 2012, vol. 4, no 4.

${ }^{4}$ Véase Aguiló, Josep: "Presunciones, verdad y normas procesales", en ISEGORÍA, núm. 35, 2006, p. 11.
} 
Valenzuela - Inocencia y razonamiento probatorio

El argumento se desarrollará en dos fases. Me referiré a la PI como regla de prueba, e intentaré mostrar cómo la PI en tanto regla de prueba en realidad es una regla que permite distribuir la carga de producir prueba (y no necesariamente la carga de persuadir mediante la prueba).

Con esto quiero decir que la pretendida garantía contenida en la PI solo abarca a una parte de la carga de la prueba. Ello necesariamente nos obliga a ver a la PI en tanto regla de prueba como una regla dirigida a las partes (al acusador), en orden a considerar por defecto probada la hipótesis que afirma la inocencia del acusado.

En la segunda fase me referiré a la PI como regla de juicio. La PI como regla de juicio supone la aplicación de un determinado estándar de prueba que permita el control intersubjetivo de los argumentos presentes en la decisión judicial de absolución o condena. En otras palabras, la garantía consiste en el uso del estándar al momento de enfrentar la PI en tanto regla de prueba. En esta medida, la PI en tanto regla de juicio tan solo es una garantía en su calidad de regla destinada al juez que supone el uso de un determinado estándar de prueba (pero que no nos dice nada acerca de ese estándar).

Finalmente, y a modo de conclusión, intentaré despejar un error a mi juicio tradicional en la conceptualización de la PI y que queda sugerido a propósito del enfrentamiento entre PI y razonamiento probatorio (es decir, en cuanto se considera a la PI en sus caras de PI-regla de prueba y PI-regla de juicio): la inocencia tiene y debe tener en el contexto de la decisión judicial una dimensión probatoria y no material.

\section{Presunción de inocencia como regla probatoria}

Como se ha tratado bastamente en la literatura, la PI tiene una clara función probatoria. Tal como todas las presunciones en el derecho, parece ser que la existencia de la PI "invierte la carga de la prueba", esto es, pone de cargo de una de las partes la necesidad de "destruir" el estado de cosas en que la presunción pone a los hechos. Se dará por existente un hecho (aunque no haya existido) si existe en su favor una determinada presunción. Es un hecho presumido de acuerdo con nuestro sencillo esquema recién expuesto.

La PI tiene una dimensión en tanto regla de prueba que, en principio, parece no escaparse de esta noción general propia de las reglas que establecen presunciones. ${ }^{5}$

\footnotetext{
${ }^{5}$ Sobre esto puede verse Ferrer, Jordi: "Una concepción..." (nota 3): Valenzuela, Jonatan:

"Presumir responsabilidad..." (nota 1).
} 
Tal como ha sostenido Ferrer al respecto: "la derrota de la presunción de inocencia debe cumplir una serie de requisitos procedimentales probatorios para que la presunción sea, efectivamente, vencida y no violada: A) debe ser la conclusión de un procedimiento en el que haya existido una mínima actividad probatoria; B) que pueda considerarse como prueba de cargo; C) suministrada por la acusación (en el sentido de que a ella corresponde la carga de la prueba); D) practicada en el juicio oral; y E) que haya sido obtenida respetando todas las garantías constitucionales y legales."

En este sentido, la PI-regla de prueba se relaciona con la actividad probatoria en el sentido de carga de producción de prueba. En el caso chileno, esta relación se produce entre la PI-regla de prueba en tanto proyección de la PI (artículo 4 del CPP) y entre el estándar de prueba contenido en el artículo 340 del CPP.

La garantía aquí parece ser redactada como sigue: "debido a que se presume inocente al imputado debemos generar prueba para desvirtuar esta situación". Y esta prueba debe ser considerada prueba de cargo, debe ser producida en el juicio oral y debe haber sido obtenida sin violación de garantías.

La PI-regla de prueba supone, necesariamente, una estrategia de disolución de una incertidumbre de hecho: entendemos decidido considerar cierto que el imputado es inocente si no es culpable. Esto quiere decir que en el caso de que un acusador pretenda que se condene a un imputado sin haber producido prueba aunque sea mínima, o que habiéndose producido prueba esta no sea considerada de cargo, o que se ha pretendido introducir el "razonamiento probatorio" en una etapa distinta del juicio oral o se han violado garantías fundamentales en la obtención de los medios de prueba, nos encontramos ante una posible violación de la garantía de la PI en su proyección de regla de prueba.

Lo importante es que la PI-regla de prueba es una regla dirigida a la selección de hechos para la decisión. Es decir, es una regla dirigida fundamentalmente a las partes, en el proceso penal a la parte acusadora, que sostiene que en caso de incertidumbre fáctica consideraremos al acusado inocente. En este sentido, lo que se busca es la toma de decisión con mayor información posible. Es decir, se busca provocar la actividad del acusador en orden a producir prueba con el fin de dar cumplimiento al doble objetivo del proceso:

"Finalidad que, a su vez, se divide en la necesidad de una doble garantía: la principal, consistente en asegurar que todos los infractores del derecho sean sancionados y que solo ellos lo sean".

\footnotetext{
${ }^{6}$ Ibid. p. 12.

${ }^{7}$ Ferrer, Jordi: “Una concepción...” (nota 3).
} 
Valenzuela - Inocencia y razonamiento probatorio

La PI-regla de prueba es una parte de la regla de distribución de cargas en materia de prueba penal, ya que lo que se encuentra imbricado es el dilema del castigo del inocente. Debemos dar por establecida una decisión en orden a que en caso de presencia de incertidumbre preferiremos dar por inocente al acusado. En algún sentido, la PI-regla de prueba es una regla de distribución de la carga de producir prueba que permite cerrar la posibilidad de encontrarnos en un caso de incertidumbre fáctica de cara a tomar una decisión de absolución o condena.

Esta sencilla operación descarta una serie de argumentos en virtud de los cuales existe algo así como "aplicación de principios", o por los cuales se haría "patente de manera directa una garantía", entre otros.

Lo que aquí ocurre es que la noción de "valoración de la prueba" (asociada a una noción opaca de "libertad de prueba" en nuestro sistema) queda entonces limitada en el momento de lo que se ha denominado "valoración estricta", esto es, a un ejercicio de corroboración entre información acerca de los hechos que dan pie a las proposiciones acerca de los mismos en el proceso. ${ }^{8}$

En este punto tenemos que se despliegan reglas acerca de la carga de la prueba, es decir, reglas que determinan quiénes deben actuar en orden a disolver la regla acerca de la incertidumbre que tenemos en el proceso (PI-regla de prueba).

\section{La presunción de inocencia como regla de juicio}

La PI no acaba de proyectarse en la regla que denominados PI-regla de prueba. Un escenario completamente diferente es el de la PI como regla de juicio en la que encontramos el momento de aplicación del estándar de prueba y la decisión de condena o absolución justificada en el cumplimiento del estándar. La PI-regla de juicio nos conduce al momento de: " $[L]$ a valoración de la prueba, de modo que si la prueba obrante en autos no resulta concluyente para demostrar la culpabilidad del imputado, la duda se resuelva a favor de la inocencia de este. Esta, por otra parte, es la tradicional lectura de la presunción de inocencia en la doctrina anglosajona.",

La PI-regla de prueba es una regla dirigida principalmente a las partes, pues define la manera en que se despliega la carga de producción de prueba, la PI-regla de juicio es una regla dirigida principalmente al juez, y dice relación con la concurrencia de la aplicación de una regla probatoria (el estándar de prueba sobre las reglas de valoración de la prueba).

\footnotetext{
${ }^{8}$ Véase Accatino, Daniela: “Certezas, dudas y propuestas en torno al estándar de la prueba penal”, en Revista de Derecho de la Ponitificia Universidad Católica de Valparaíso, núm. XXXVII, 2011, pp. 483-511.

${ }^{9}$ Ferrer, Jordi: "Una concepción...” (nota 3).
} 
Lo que debe anotarse es que mediante la PI-regla de juicio se da por supuesta la aplicación de un determinado estándar, sin embargo, nada se dice acerca de la forma y contenido de este estándar.

La PI-regla de juicio nos conduce a sostener que, en realidad, las cuestiones acerca de qué estándar (qué clase de distribución de errores) está reservada a la etapa anterior, en la que el juez trabaja con PI-regla de prueba.

Con esto quiero decir que podría darse el caso en el que aun sosteniendo una tesis acerca de las proyecciones probatorias de la PI (como la que intento sostener aquí) tuviéramos un estándar de prueba subjetivo (es decir, un estándar de prueba en realidad aparente)..$^{10}$

Es, de todos modos, importante poder distinguir ambos momentos de proyección de la PI (PI-regla de prueba y PI-regla de juicio), pues de esta manera podemos diferenciar las cuestiones asociadas a la carga de la prueba y las cuestiones que se relacionan con la valoración de la misma.

La PI-regla de juicio supone el aseguramiento por parte del juez de haber vencido por un medio institucional determinado a la PI-regla de prueba.

A esto es, en mi opinión, a lo que apela nuestro CPP en las letras b y c del artículo 342 que señala que la sentencia debe contener:

"b) La enunciación breve de los hechos y circunstancias que hubieren sido objeto de la acusación; en su caso, los daños cuya reparación reclamare en la demanda civil y su pretensión reparatoria, y las defensas del acusado; c) La exposición clara, lógica y completa de cada uno de los hechos y circunstancias que se dieren por probados, fueren ellos favorables o desfavorables al acusado, y de la valoración de los medios de prueba que fundamentaren dichas conclusiones de acuerdo con lo dispuesto en el artículo 297”.

Esta exigencia permite dar por concurrente un razonamiento probatorio controlable. Es decir, las proposiciones acerca de los hechos contenidas en estos numerales del artículo 342 nos lleva a dar por cumplidos criterios de aplicación de las reglas de valoración de la prueba y parece ser posible considerar además concurrente la aplicación de un determinado estándar de prueba que se someta al control derivado de su vinculación a la claridad, la lógica y la plenitud.

\footnotetext{
${ }^{10}$ Aunque es evidente que existen buenas razones para descartar un estándar de prueba meramente subjetivo. Sobre el particular puede verse el trabajo de Laudan, Larry: "Por qué un estándar de prueba subjetivo y ambiguo no es un estándar", en Doxa: Cuadernos de Filosofía del Derecho, núm. 28, 2005.
} 
Valenzuela - Inocencia y razonamiento probatorio

\section{Un estándar que incluya o denote a PI-regla de prueba y a PI-regla de juicio}

Ambas proyecciones probatorias de la PI nos llevan a tematizar el tipo de estándar de prueba que resulte más compatible con su conceptualización. Creo, naturalmente, que debe tratarse de un estándar de prueba racional que permita el control intersubjetivo de la decisión.

Un claro intento de formulación de un estándar de prueba (alto) ha sido ofrecido en repetidas ocasiones por Ferrer:

"Imaginemos que nuestra ley de enjuiciamiento criminal estableciera que para considerar probada la hipótesis de la culpabilidad deben darse conjuntamente las siguientes condiciones: 1) La hipótesis debe ser capaz de explicar los datos disponibles, integrándolos de forma coherente, y las predicciones de nuevos datos que la hipótesis permita formular deben haber resultado confirmadas.

2) Deben haberse refutado todas las demás hipótesis plausibles explicativas de los mismos datos que sean compatibles con la inocencia del acusado, excluidas las meras hipótesis ad hoc."

Esta es una manera de redactar un estándar con bastante precisión y que conlleva a mostrar nuestro punto. La PI se proyecta en dos reglas principales: la PI-regla de prueba que impacta en la entrega de datos probatorios que sirven de base para tener hipótesis, y si estos datos no existen o son insuficientes no se permite una incertidumbre fáctica, sino que se da por existente la hipótesis de la inocencia.

Por otra parte, la PI proyectada en PI-regla de juicio da cuenta de la valoración de la prueba, en tanto regla que supone un estándar. Es simplemente una manera de decir que existe una hipótesis que ha dejado satisfecha la premisa del estándar, pero no prejuzga la forma ni el contenido del mismo.

Lo llamativo es que en este punto es donde suele desplegarse la denominada garantía denominada in dubio pro reo. Esto a mi juicio es una confusión debido a que en realidad lo que denominamos in dubio pro reo no es sino una limitación acerca de la negativa de incertidumbre que conlleva la existencia de la PI-regla de prueba y PI-regla de juicio.

¿Qué clase de estándar es el que razonablemente deja satisfechos los requisitos de la PI? Diríamos que cualquier estándar que se alinee con los requerimientos ya vistos acerca de la racionalidad de la decisión judicial.

\footnotetext{
${ }^{11}$ Ferrer, Jordi: “Una concepción...” (nota 3).
} 
En este sentido, cabe notar que tan solo un estándar entendido como una herramienta de distribución de errores que sea intersubjetivamente controlable puede dar verdadero sentido a la PI en tanto garantía en el contexto probatorio. Esto resulta obvio, atendido que el sentido de la PI no es en caso alguno tributario de una noción material de inocencia (o al menos no lo es en términos de operación). ${ }^{12}$

\section{A modo de conclusión: inocencia en términos probatorios}

Una interesante forma de dar proyección a nuestro problema ha sido tocado por Laudan: ¿cuál es el sentido en el que utilizamos la expresión inocencia? ${ }^{13}$

El propio Laudan advierte dos vías para conceptualizar a la inocencia: esta puede ser o bien material o bien probatoria. ${ }^{14}$

La inocencia en sentido material se encuentra referida al hecho simple de la no realización del delito por parte del imputado.

En cambio, la inocencia en términos probatorios nos dice que respecto de un determinado imputado no se ha producido prueba que iguale o supere el estándar establecido en el proceso para que sea considerado culpable.

Para Laudan lo interesante es contraponer los sentidos material y probatorio de la inocencia con los sentidos material y probatorio de la culpabilidad.

Un imputado puede ser materialmente culpable. Este es el caso en que el imputado o acusado haya en realidad cometido los hechos materia de la acusación. Pero, por otra parte, un imputado o acusado puede ser probatoriamente culpable. Es decir, puede haberse superado respecto de su acusación el estándar de prueba que le señala como culpable, pero en realidad no ha cometido los hechos por los cuales se le acusa.

Si quisiéramos graficar o resumir las alternativas que tenemos:

Inocente $(\mathrm{m})[1]$ - Inocente(p)[2] vs culpable (m)[3] - culpable (p)[4]

El problema apuntado por Laudan se produce cuando tenemos a un inocente probatorio que es a la vez culpable material; es decir, cuando tenemos la hipótesis $[2] \wedge[3]$.

\footnotetext{
12 Sobre el particular véase Laudan, Larry: "Por qué un estándar..." (nota 10).

${ }^{13}$ Véase Laudan, Larry: "The presumption of innocence: Material or Probatory?", en Legal Theory, núm 11, 2005, pp. 333-361.

${ }^{14}$ Ibid. p. 342.
} 
Valenzuela - Inocencia y razonamiento probatorio

El punto central a considerar es que, a efectos del razonamiento probatorio, la noción de inocencia que debe manejarse se encuentra referida necesariamente a la proyección de la PI en PI-regla de prueba y PI-regla de juicio, con lo que es claro que trabajamos con una noción probatoria de inocencia (o sea, [2] y nunca [1]).

Por lo mismo es evidente que siempre nos referimos a un culpable en términos también probatorios, que puede concurrir lógicamente con el culpable en términos materiales, pero cuya concurrencia es resultado de un correcto trabajo procesal. En este sentido, la manera de graficar o resumir las alternativas sería:

$\{$ Inocente(m)[1]\}- Inocente(p)[2] vs Culpable(p)[4]VCulpable(m)[3]

Con esto no pretendo sostener que la inocencia en términos materiales, es decir, el hecho de que el sujeto no haya cometido el delito efectivamente sea irrelevante en el proceso, tan solo es un dato inalcanzable en el contexto del diseño del razonamiento acerca de la prueba en el proceso penal.

Esto es razonable debido a que en el proceso y en particular en las instituciones probatorias de lo que se trata es de repartir el error que inevitablemente se producirá: se condenará a culpables o se absolverá a inocentes. Nuestro estándar de prueba ("más allá de toda duda razonable") constituye un modo de distribución de estos errores, reduciendo la posibilidad de condenar a inocentes y aceptando en mayor medida la posibilidad de absolver a culpables.

Este es el núcleo central de la PI en el contexto del razonamiento probatorio: es una manera de tomar partido en el caso de incertidumbre fáctica en el proceso a favor de la absolución de culpables o en contra de la condena de inocentes. 
REJ - Revista de Estudios de la Justicia - No 18 - Año 2013

\section{BIBLIOGRAFÍA}

* ACCATINO, Daniela: "Certezas, dudas y propuestas en torno al estándar de la prueba penal", en Revista de Derecho de la Ponitificia Universidad Católica de Valparaíso 2011, núm. XXXVII,

* AGUILÓ, Josep: "Presunciones, verdad y normas procesales", en Isegoría, núm. 35, 2006.

* BOHLEN, Francis: "The Effect of Rebuttable Presumptions of Law upon the Burden of Proof", en University of Pennsylvania Law Review and American Law Register, vol. 68, núm. 4, 1920.

* FERRER, Jordi: "Una concepción minimalista y garantista de la presunción de inocencia", inédito, 2011.

* LAUDAN, Larry: "Por qué un estándar de prueba subjetivo y ambiguo no es un estándar", en Doxa: Cuadernos de Filosofía del Derecho, núm. 28, 2005. 2005.

"The presumption of innocence: Material or Probatory?”, en Legal Theory, núm 11,

* VALENZUELA, Jonatan: "Presumir responsabilidad: sobre una relación entre la prisión preventiva y la presunción de inocencia en el derecho procesal chileno", en Revista de Estudios de la Justicia, núm. 14, 2011.

* YABLON, Charles: "A Theory of Presumptions", en Law, Probability and Risk, núm. 2, 2003. 